\title{
Molecular Typing by Polymerase Chain Reaction Sequence Specific Primers (PCR- SSP) of Human Leukocyte Class I and Class II Alleles in a Sample of Iraqi Visceral Leishmaniasis Patients
}

\begin{abstract}
Nidal Abdul Mohymen ${ }^{1}$, Abdullah M. Qader ${ }^{2}$ and Ali H. Ad'hiah ${ }^{3}$
1- Department of Microbiology, College of Medicine, Al-Nahrain University, Baghdad, Iraq

dr.nidhalmohammed@yahoo.com

2- Biotechnology Research Center, Al-Nahrain University, Baghdad, Iraq.

haddad_biotech@yahoo.com

3- Tropical-Biological Research Unit, College of Science, University of Baghdad, Baghdad, Iraq.

Adhiah1756@yahoo.com

Objectives: This study aimed to investigate the association between HLA alleles and visceral leishmaniasis (VL) in a sample of Iraqi patients. Methods: A total of 30 patients were studied, in addition to 20 age, gender and ethnicity matched controls. All subjects were genotyped by polymerase chain reaction-sequence specific primers (PCR-SSP) method. Results: For HLA-class I region ( $A$ and $B$ loci), only HLA-A*19 allele showed a significant $(P=0.031)$ decreased frequency in VL patients as compared with controls (13.3 vs. $45.0 \%$ ), and such deviation was associated with OR and PF values of 0.19 and 0.37 , respectively. At HLA-class II region, HLA-DRB ${ }^{*} 03$ and HLA-DQB1*02 alleles were significantly $(P=0.020$ and 0.013 , respectively) increased in VL patients (56.6 vs. $20 \%$ and 46.6 vs. $10 \%$, respectively) as compared with controls. The OR of such two positive associations was 5.23 and 7.88 , respectively, and the EF value was 0.46 and 0.41 , respectively. In contrast, HLA-DRB1*02 (13.3 vs. $45.0 \%)$ and HLA-DQB1*03 (33.3 vs. $70.0 \%)$ were significantly $(P=$ 0.031 and 0.023 , respectively) decreased in patients. However, none of these differences remained significant after correcting the $P$ value for the number of alleles tested at each locus. Conclusion: these preliminary data suggest that HLA alleles may have some role in aetiopathogenesis of VL, and this role can be in favour of susceptibility and/or protection.
\end{abstract}

ABSTRACT

\section{Indexing terms/Keywords}

HLA; PCR; Visceral leishmaniasis.

\section{Academic Discipline And Sub-Disciplines} Immunogenetics.

\section{Subject Classification}

Immunology.

\section{Type (Method/Approach)}

Quasi-Experimental.

\section{Council for Innovative Research}

Peer Review Research Publishing System

\section{Journal: JOURNAL OF ADVANCES IN BIOTECHNOLOGY}

Vol .4, No 1.

www.ciribt.org , jbteditor@gmail.com 


\section{INTRODUCTION}

Visceral leishmaniasis (VL) is a human protozoal disease, which is caused by the intracellular parasites Leishmania donovani and Leishmania infantum [1]. The disease, also called Kala-azar or black fever, is prevalent throughout the world, and is recognized as an important public health problem, and in Iraq, it is the most devastating and fatal form [2]. Factors determining whether a patient remains asymptomatic or develops VL are still largely unknown; however, host genetic and immunological factors are considered as one of the main determinants that influence the susceptibility to develop the disease. Both factors are better understood in the ground of human major histocompatibility complex (MHC); the HLA system [3,4]. The system is controlled by polymorphic genes located on the short arm of chromosome 6 , and a cellular expression of their products is presented through two classes of antigens; class I (A, B and C) and class II (DR, $\mathrm{DQ}$ and DP), whose function is to present antigenic peptides to CD4+ and CD8+ T cell, and therefore, they play a fundamental role in various cellular interactions required during the immune response [5].

A host immune reactivity is held responsible for the pathological evolution of infectious diseases; moreover, such reactivity has been either positively or negatively associated with alleles of HLA system, including VL, in which the studies have demonstrated associations between the disease and specificities of HLA system, especially at the serological level [6]. With the development of HLA molecular typing, the HLA association with VL and other parasitic infections can be better understood in terms of a genetic predisposition and may facilitate the development of vaccines for these infectious diseases [7]. Accordingly, the present study was undertaken to investigate the association of HLA class I (A and B) and class II (DR and DQB) alleles with VL in a sample of Iraqi patients, using a DNA based HLA typing method.

\section{SUBJECTS AND METHODS}

Blood samples were obtained from 30 unrelated, parasitologically proven VL patients (12 males and 18 females) undergoing treatment at three hospitals in Baghdad during the period October 2005 - May 2006. All cases were hospitalized patients, and their age range was 5 months to 12 years at the time of admission. Patient evaluation included history and physical examination by the consultant medical staff at the hospitals. All patients experienced subacute onset of fever, weakness, fatigue, weight loss, splenomegaly and hepatomegaly. Laboratory findings included anemia, leucopenia, and hypergammaglobulinemia. Furthermore, the disease was confirmed by determination of anti-leishmania donovani IgG antibodies in the sera of patients using ELISA techniques (IVD Research Inc. Carlsbad, CA92008 USA). Twenty individuals, gender, age and ethinicity matched, from the same endemic area were also included as healthy controls.

Genomic DNA was isolated from blood samples using a commercially available kit (E.Z.N.A. RSQ, Omega Bio-teck). All samples were genotyped for four HLA genes (A, B, DRB1 and DQB1) by polymerase chain reaction-sequence specific primers (PCR-SSP), which were available as a ready used kit (HISTOTYPE/DNA-SSP-PAG-Germany). The laboratory procedures of such manipulations were followed according to the manufacturers of kits.

The results are presented as percentage frequencies of alleles, and differences between patients and controls were assessed by two-sided Fisher's exact probability $(P)$, and the $P$ value was corrected for the number of alleles tested at each locus $(\mathrm{Pc})$. Alleles showing a significant variation were assessed fo odd's ratio $(\mathrm{OR})$, etiological fraction (EF) and preventive fraction (PF). Furthermore, the Fisher test power (FTP) and 95\% Fisher's interval (95\% FI) were also given [8]. Such analyses were carried out using the computer programme PEPI version 4.0.

\section{RESULTS}

For HLA-class I region ( $A$ and $B$ loci), only HLA-A*19 allele showed a significant $(P=0.031)$ decreased frequency in VL patients as compared with controls (13.3 vs. $45.0 \%)$, and such deviation was associated with OR and PF values of 0.19 and 0.37 , respectively. At HLA-class II region, HLA-DRB1*03 and HLA-DQB1*02 alleles were significantly $(P=0.020$ and 0.013 , respectively) increased in VL patients ( 56.6 vs. $20 \%$ and 46.6 vs. $10 \%$, respectively) as compared with controls. The OR of such two positive associations was 5.23 and 7.88 , respectively, and the EF value was 0.46 and 0.41 , respectively. In contrast, HLA-DRB1*02 (13.3 vs. $45.0 \%)$ and $\mathrm{HLA}-\mathrm{DQB} 1{ }^{*} 03(33.3$ vs. $70.0 \%)$ were significantly $(\mathrm{P}=$ 0.031 and 0.023 , respectively) decreased in patients. However, none of these differences remained significant after correcting the $P$ value for the number of alleles tested at each locus (Table 1).

Table 1. HLA alleles showing variations between VL patients and controls.

\begin{tabular}{|c|c|c|c|c|c|c|c|c|c|c|}
\hline \multirow[t]{2}{*}{ HLA Alleles } & \multicolumn{2}{|c|}{$\begin{array}{l}\text { Patients } \\
(\text { No. }=30)\end{array}$} & \multicolumn{2}{|c|}{$\begin{array}{l}\text { Controls } \\
(\text { No. }=20)\end{array}$} & \multirow[t]{2}{*}{ OR } & \multirow{2}{*}{$\begin{array}{l}\text { EF } \\
\text { or } \\
\text { PF }\end{array}$} & \multirow[t]{2}{*}{$\mathbf{P}$} & \multirow[t]{2}{*}{ Pc } & \multirow{2}{*}{$\begin{array}{l}\text { FTP } \\
\text { (\%) }\end{array}$} & \multirow[t]{2}{*}{$95 \% \mathrm{FI}$} \\
\hline & No. & $\%$ & No. & $\%$ & & & & & & \\
\hline HLA-A*19 & 4 & 13.3 & 9 & 45.0 & 0.19 & 0.37 & 0.031 & NS & 66.6 & $0.036-0.880$ \\
\hline HLA-DRB $1{ }^{*} 02$ & 4 & 13.3 & 9 & 45.0 & 0.19 & 0.37 & 0.031 & NS & 66.6 & $0.036-0.880$ \\
\hline HLA-DRB $1{ }^{*} 03$ & 17 & 56.6 & 4 & 20.0 & 5.23 & 0.46 & 0.020 & NS & 66.2 & $1.231-25.902$ \\
\hline HLA-DQB $1{ }^{*} 02$ & 14 & 46.6 & 2 & 10.0 & 7.88 & 0.41 & 0.013 & NS & 70.0 & $1.403-78.815$ \\
\hline HLA-DQB1*03 & 10 & 33.3 & 14 & 70.0 & 0.21 & 0.20 & 0.020 & NS & 65.0 & $0.052-0.839$ \\
\hline
\end{tabular}


OR: Odd's Ratio; EF: Etiological Fraction; PF: Preventive Fraction; P: Two-sided Fisher's Exact Probability; Pc: Corrected P; FTP: Fisher test power; 95\% FI: 95\% Fisher's interval; NS: Not significant.

\section{DISCUSSION}

The present study demonstrated that immunogenetic predisposition may be considered as an important requirement for the development of $V L$, and $H L A$ alleles are in favour of such generalization, in which five alleles (HLA-A*19, HLADRB1*02, HLA-DRB1*03, HLA-DQB1*02 and HLA-DQB1*03) of human MHC showed different distributions in patients and controls, but the significance of such variations was lost when it was corrected. This may be reasoned by the fact of a small sample size in patients or controls. At HLA-class I region, a decreased frequency of HLA-A*19 was observed in VL patients compared with controls, and such deviation scored a PF value of 0.37 . This result is not in agreement with other studies conducted in India [9], Tunisia [10], and Brazil [11], as well as, a regional study carried out in Iran [12]. The latter study demonstrated that A26 was significantly increased, but their typing was at the serological level. Inspecting other HLA-VL association studies carried out in other world populations revealed associations with other HLA- class I alleles [13-15]. Such discrepancy can be explained in the ground of racial differences, especially if we consider that HLA antigens show different frequencies in different populations including Iraqis [16,17]. Therefore, the discrepancy between the present study and other world studies can be ascribed to either racial differences (different associations in different populations), low sample size (the level of significance is affected) or environmental impacts (different Leishmania strains). With respect to the latter factor, the phenotypic expression of HLA-class II antigens is also involved in antigen presentation by macrophages, but with antigens of external origin (e.g. parasite), and such pathogen may have adapted different epitopes in different populations $(6,7,18)$. Moreover, in the present study, a DNA based HLA typing method was employed, namely PCR-SSP, which may help to improve the accuracy of HLA typing and a better theme of MHC-disease association can be pictured.

At HLA-class II region, two alleles were observed with an increased frequency (HLA-DRB1*03 and HLA-DQB $\left.{ }^{*} 02\right)$ in the patients, while further two alleles demonstrated a decreased frequency (HLA-DRB1*02 and HLA-DQB1*03). Such observations may suggest that the former alleles are associated with an increased risk to develop the disease $(\mathrm{OR}=5.23$ and 7.88, respectively), while the latter two alleles may be considered as protected factors. Such findings have some support from two previous studies [10,19], in which DR2 was associated with a greater protection against the disease. Furthermore, a study carried out in Mexico reported that DQ3 antigen plays some role in host susceptibility to the infection and DR2 has a significant role for protection [20]. However, further two studies carried out in Brazil [11] and India [9] failed to report similar findings, but a further Indian study demonstrated that a greater expression of HLA-DR antigens is associated with activation of $T$ cells in the immune response against infection with L. donovani [21].

In conclusion, these preliminary data suggest that HLA alleles may have some role in the aetiopathogenesis of VL, and this role can be in favour of susceptibility and/or protection, but the results must be interpreted with caution due to small size, and certainly, further studies are required to shed light on these associations, especially if the results are interpreted in terms of parasite stains, as well as, other humoral and cellular components of the immune response.

\section{REFERENCES}

[1] Palumbo, E. (2010). Visceral leishmaniasis in children: a review. Minerva Pediatr, 62: 389-395.

[2] Postigo, J.A. (2010). Leishmaniasis in the World Health Organization Eastern Mediterranean Region. Int J Antimicrob Agents, 36 Suppl 1: S62-S65.

[3] Sakthianandeswaren, A., Foote, S.J. and Handman, E. (2009). The role of host genetics in leishmaniasis. Trends Parasitol, 25: 383-391.

[4] Sharma, U. and Singh, S. (2009). Immunobiology of leishmaniasis. Indian J Exp Biol, 47: 412-423.

[5] Howell, W.M., Carter, V. and Clark, B. (2010). The HLA system: immunobiology, HLA typing, antibody screening and crossmatching techniques. J Clin Pathol, 63: 387-390.

[6] Alves, C., Souza, T., Meyer, I., Toralles, M.B. and Brites, C. (2006). Immunogenetics and infectious diseases: special reference to the major histocompatibility complex. Braz J Infect Dis, 10: 122-131.

[7] Blackwell, J.M., Jamieson, S.E. and Burgner, D. (2009). HLA and infectious diseases. Clin Microbiol Rev, 22: $370-$ 385.

[8] Tevfik Dorak, M. (2009). Statistical analysis in HLA and disease association studies. Available at http://www.dorak.info/hla/stat.html (Updated 27 July 2009).

[9] Singh, N., Shyam, S., Fionnuala, W., Martin, D., Curran Anill, R. et al. (1997). Molecular typing of HLA class I and class II antigens in Indian Kala-azar patients. Trop Med Intern Heal, 2: 468-471.

[10] Meddeb-Garnaoui, A., Gritli, S., Garbouj, S., Ben Fadhel, M., El Kares, R., Mansour, L. et al. (2001). Association analysis of HLA-class II and class III gene polymorphisms in the susceptibility to Mediterranean visceral leishmaniasis. Hum Immunol, 62: 509-517. 
[11] Peacock, C.S., Sanjeevi, C.B., Shaw, M.A., Collins, A., Campbell, R.D., March, R. et al. (2002). Genetic analysis of multicase families of visceral leishmaniasis in northeastern Brazil: no major role for class II or class III regions of HLA. Genes Immun, 3: 350-358.

[12] Faghiri, Z., Tabei, S.Z. and Taheri, F. (1995). Study of the association of HLA class I antigens with Kala-azar. Hum Hered, 45: 258-261.

[13] Morsy, T.A., Romia, S.A., Al-Ganayni, G.A., Abu-Zakham, A.A., Al-Shazly, A.M. and Rezk, R.A. (1990). Histocompatibility antigens (HLA) in Egyptians with two parasitic skin diseases (scabies and leishmaniasis). J Egypt Soc Parasitol, 20: 565-572.

[14] Lara, M.L., Layrisse, Z., Scorza, J.V., Garcia, E., Stoikow, Z., Granados, S. et al. (1991). Immunogenetics of human American cutaneous leishmaniasis. Study of HLA haplotypes in 24 families from Venezuela. Hum Immunol, 30: 129-135.

[15] Ameen, M. (2009). Cutaneous leishmaniasis: disease susceptibility and pharmacogenetic implications. Pharmacogenomics, 10: 451-461.

[16] Ad'hiah A.H. (1990). Immunonogenetic studies in selected human diseases. Ph.D. Thesis, Department of Human Genetics, 1990; University of Newcastle upon Tyne. U.K.

[17] Ad'hiah, A.H. (2009). Distribution of HLA polymorphism in a sample of Iraqi Arabs in comparison with three Arab Gulf populations. Iraqi Journal of Science, 50: 120-125.

[18] Handman, E., Elso, C. and Foote, S. (2005). Genes and susceptibility to leishmaniasis. Adv Parasitol, 59 : 1-75.

[19] Petzl-Erler, M.L., Belich, M.P. and Queiroz-Telles, F. (1991). Association of mucosal leishmaniasis with HLA. Hum Immunol, 32: 254-260.

[20] Olivo-Díaz, A., Debaz, H., Alaez, C., Islas, V.J., Pérez-Pérez, H., Hobart, O. et al. (2004). Role of HLA class II alleles in susceptibility to and protection from localized cutaneous leishmaniasis. Hum Immunol, 65: 255-261.

[21] Lal, S., Bimal, S., Sinha, A.N. and Prasad, LS. (1991). Role of HLA-DR antigens on T-cell activation in visceral leishmaniasis. Indian J Exp Biol, 29: 1101-1103. 